\title{
Advances in the drug therapies of acute myeloid leukemia (except acute promyelocytic leukemia)
}

This article was published in the following Dove Press journal:

Drug Design, Development and Therapy

Min Lin

\section{Baoan Chen}

Department of Hematology and Oncology, Zhongda Hospital, School of Medicine, Southeast University, Nanjing, People's Republic of China
Correspondence: Baoan Chen Department of Hematology and Oncology, Zhongda Hospital, School of Medicine, Southeast University, 87 Dingjiaqiao, Gulou, Nanjing 210009 , Jiangsu, People's Republic of China Email cba8888@hotmail.com

\begin{abstract}
Acute myeloid leukemia (AML) is a heterogeneous hematologic malignancy, characterized by the clonal expansion of myeloid blasts in the peripheral blood, bone marrow, and/or other tissues. The new drugs used for treating AML are facing a big challenge, and the candidates include cytotoxic drugs, targeted small-molecule inhibitors, and monoclonal antibodies. In recent years, active research has focused on several new agents for including them in the large antileukemic drug family. This review aims to introduce some of these new drugs and highlights new advances made in the old drugs, mainly in the last 5 years.
\end{abstract}

Keywords: acute myeloid leukemia, new drug therapies, refractory/relapsed AML, 2017 NCCN guideline

\section{Introduction}

Acute myeloid leukemia (AML), a heterogeneous hematologic malignancy, is characterized by the clonal expansion of myeloid blasts in the peripheral blood, bone marrow, and/or other tissues. AML has the highest incidence compared to other acute leukemias (ALs) and also the highest mortality rate among the diseases of the hematologic system. It is estimated that 5,970 people will be newly diagnosed with AML in 2017, and 1,440 patients will die of this disease in the USA. ${ }^{1}$ Its treatment difficulties are mainly reflected in resistance to drugs, including primary resistance, which exists before the treatment, and secondary resistance, which develops due to the repeated use of some induction chemotherapeutic agents. This directly leads to refractory and/or relapsed AML and reduces the overall survival (OS) of AML patients. There are several approaches to the treatment of AML, which include chemotherapy, targeted therapy, immunotherapy, and hematopoietic stem cell transplantation (HSCT).

For this review, we conducted a literature search of the studies on drug therapies (except HSCT) used for AML, especially those published in the last 5 years.

\section{Chemotherapy}

Standard induction regimens from the National Comprehensive Cancer Network (NCCN) guidelines (V3.2017) and their new advances

The NCCN guidelines (V3.2017)2 mainly recommend 5 kinds of induction regimens based on history of prior myelodysplasia or cytotoxic therapy, age, and performance status. First, the standard induction regimen, which is well known as " $7+3$ " induction therapy, used for patients younger than 60 years, has changed a little in the past 25 years. The regimen involves infusion of anthracycline (daunorubicin [Pfizer, USA] 45-60 mg/m² daily or idarubicin [Jian An, China] $12 \mathrm{mg} / \mathrm{m}^{2}$ daily) for 3 days, and

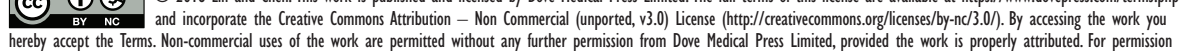
hor commercial use of this work, please see paragraphs 4.2 and 5 of our Terms (https://www.doveppess. com/terms.php).
for 
cytarabine (Pfizer, USA) for 7 days via continuous infusion (100-200 mg/m² daily), which is still the standard induction therapy for adult patients. In recent years, there is increased focus on the dose of anthracycline. Padron and Fernandez ${ }^{3}$ reported a significant increase of complete response (CR) rates $(70.6 \%$ versus $57.3 \%, p<0.001)$ and OS (median 23.7 versus 15.7 months, $p=0.003$ ) using daunorubicin $90 \mathrm{mg} / \mathrm{m}^{2}$ versus $45 \mathrm{mg} / \mathrm{m}^{2}$ in patients younger than 60 years old with favorable- or intermediate-risk cytogenetics. The second standard regimen involves addition of cladribine $5 \mathrm{mg} / \mathrm{m}^{2}$ from day 1 to 5 to the " $7+3$ " regimen (cytarabine [Haizheng, China] $200 \mathrm{mg} / \mathrm{m}^{2}$, daunorubicin $60 \mathrm{mg} / \mathrm{m}^{2}$ ). Holowiecki et $\mathrm{al}^{4}$ conducted a Phase III study, and reported a significant improvement in the $\mathrm{CR}$ rate $(67.5 \%$ versus $56 \%, p=0.01)$ and 3 -year OS $(45 \% \pm 4 \%$ versus $33 \% \pm 4 \%)$. The third regimen recommended by the NCCN guidelines is the "HiDAC regimen" (cytarabine $3 \mathrm{~g} / \mathrm{m}^{2}$ per 12 hours via 3-hour infusion on days $1,3,5$, and 7 ; daunorubicin $60 \mathrm{mg} / \mathrm{m}^{2}$ or idarubicin $12 \mathrm{mg} / \mathrm{m}^{2}$ on days $\left.1-3\right)$, the use of which for induction therapy in younger patients is still controversial. However, its efficacy in patients younger than 46 years old has been proved by Willemze et al. ${ }^{5}$ They reported a Gruppo Italiano Malattie Ematologiche dell'Adulto (GIMEMA) and the European Organization for Research and Treatment of Cancer (EORTC) Leukemia Groups randomized trial, concluding that young adults, that is, patients who are younger than 46 years old, can achieve a significant benefit from the regimen (a median 6-year follow-up showed an OS rate of $51.9 \%$ versus the standard Decitabine, Cytarabine and G-CSF (DAC) regimen's 43.3\%, $p=0.009$ ). However, a new modified HiDAC regimen consisting of cytarabine $2 \mathrm{~g} / \mathrm{m}^{2}$ for 6 days in combination with daunorubicin $45 \mathrm{mg} / \mathrm{m}^{2}$ for 3 days was applied in 59 consecutive patients older than 60 years old who had de novo AML diagnosed between July 1996 and February 2005. The results showed that the modified regimen was well tolerated and $69 \%$ of patients achieved CR, and $80 \%$ received HiDAC for 3 consolidations. The median OS of patients was 15.3 months, and the relapse-free survival (RFS) was 13.8 months. Survival of patients who achieved CR was 27 months and ranged from 2 to 114 months. ${ }^{6}$ New "FLAGIDA" regimen is the fourth effective induction regimen with a high CR rate introduced by the NCCN guidelines. This consists of infusion of fludarabine $30 \mathrm{mg} / \mathrm{m}^{2}$ (Bayer, Germany) on days 2-6; 4 hours after fludarabine, cytarabine $2 \mathrm{~g} / \mathrm{m}^{2}$ should be infused over 4 hours, idarubicin $8 \mathrm{mg} / \mathrm{m}^{2}$ on days 4-6, and granulocyte colony-stimulating factor (G-CSF) daily during days $1-7$. Burnett et al indicated that, after receiving 2-course FLAG-IDA (cytarabine 3 or $1.5 \mathrm{~g} / \mathrm{m}^{2}$ ), patients with intermediate risk and favorable risk achieved an 8 -year survival of $63 \%$ and $95 \%$, respectively. ${ }^{7}$ The last regimen is the lower-intensity therapy which is usually used for patients over 60 years old. Specific conditions for the use of these regimens are described below.

\section{New cytotoxic agents}

In recent years, several studies have been focusing on a third cytotoxic agent besides cytarabine and anthracycline. Monotherapy using these agents or their combination with other drugs has brought bright prospects in the therapy of AML. According to the research conducted in the last 5 years, these agents have been categorized as follows.

\section{Hypomethylating agents (HMAs)}

It has been shown that epigenetic silencing of structurally normal genes by abnormal DNA methylation, which is mediated by DNA-methyltransferase (DNMT) enzymes, leads to the myeloid leukemogenesis, especially in older patients. ${ }^{8}$ However, the underlying molecular mechanisms are still unclear. Some articles have reported that a key reason is the reactivation of silenced tumor suppressor genes, ${ }^{9}$ while others have indicated that HMAs can induce the expression of tumor antigens, like cancer testis antigens. ${ }^{10,11}$ In older patients, HMAs bring a similar outcome on intensive chemotherapy, but a lower toxicity. ${ }^{12}$ Azacitidine (5-azacitidine) (Baxter, Germany) and decitabine (5-aza-2'-deoxycitidine) (Haosen, China) are the 2 main widespread azanucleoside DNMT inhibitors, which are also recommended in the new NCCN guidelines as a lower-intensity therapy for the elderly. Researches indicated that compared with low-dose cytarabine or supportive care, they can effectively prolong the OS of the subgroups. ${ }^{13-17}$ Due to advances in research, a novel secondgeneration HMA, guadecitabine (BOC Science, USA), has come into our sight. It is a dinucleotide of deoxyguanosine and decitabine and is resistant to degradation by cytidine deaminase. Issa et al assessed the safety and clinical activity of this agent through a multicenter, open-label, Phase I study. The results showed that guadecitabine $60 \mathrm{mg} / \mathrm{m}^{2}$ for days $1-5$ is the recommended Phase II dose, and further studies are ongoing and preliminary data are awaited. ${ }^{18}$

\section{New nucleoside analogs}

Clofarabine (Genzyme, USA), a new second-generation nucleoside analog, has shown significant effect as monotherapy and in combination with multiple drugs in the treatment of AML. A Phase II study used clofarabine monotherapy in newly diagnosed older AML patients (median age 71 years, range $60-88$ years), at $30 \mathrm{mg} / \mathrm{m}^{2}$ infusion for induction on days $1-5$ and $20 \mathrm{mg} / \mathrm{m}^{2}$ during reinduction/consolidation, for 
totally 6 cycles. As a result, the overall response rate (ORR) was $46 \%$ (CR rate $38 \%$ ), and the median remission duration was 56 weeks. ${ }^{19}$ On the other hand, clofarabine plus low-dose cytarabine (CLDA) is also considered the frontline therapy for elderly patients with high response rate and tolerated toxicity. To test this combination, researchers conducted a propensity score-matched comparison of AML patients aged more than 60 years given CLDA versus idarubicin and cytarabine (IA) for induction therapy. The results showed that CLDA produced significantly fewer grade 3 or worse toxicities ( $46 \%$ for CLDA versus $62 \%$ for IA, $p=0.03$ ) and the median response duration was also longer than that of patients who underwent HSCT (15.9 months for cytarabine versus 7.0 months for IA, $p=0.033){ }^{20}$

Sapacitabine (Medical Isotopes, USA) is another novel oral cytosine nucleoside analog, which can break the DNA strands causing apoptosis..$^{21,22}$ Kantarjian et al tested the efficacy and toxicity of sapacitabine in a randomized Phase II study. They treated the patients starting with a dose of $200 \mathrm{mg}$ twice a day for 7 days, followed by $300 \mathrm{mg}$ twice a day for 7 days and then $400 \mathrm{mg}$ twice a day for 7 days. The data indicated that the $400 \mathrm{mg}$ dose schedule showed the best efficacy profile. ${ }^{23}$ Regarding clinical effects, Green et al concluded that sapacitabine plus histone deacetylase (HDAC) inhibitors is an effective combination. ${ }^{24}$ However, there is still no sufficient number of trials to prove the efficacy of sapacitabine monotherapy. Further trials on the efficacy of sapacitabine are underway.

\section{HDAC inhibitors}

Histone acetylation and deacetylation are necessary processes of gene expression regulation. Histone acetylation releases the condensed chromatin and exposes the promoter regions of genes to transcription factors, while deacetylation catalyzed by HDACs causes gene silencing. ${ }^{25}$ In leukemic cells, this balance is disrupted by several mechanisms, and HDAC inhibitors can act as an attractive therapeutic agent to modulate the disease.

Vorinostat (Medchem Express, USA) is a HDAC inhibitor approved in the USA as monotherapy but has limited activity in cutaneous T-cell lymphoma. To assess the safety and efficacy of the combination of vorinostat with the "IA regimen" in the treatment of patients with AML or myelodysplastic syndrome, researchers designed an induction therapy consisting of infusion of vorinostat $500 \mathrm{mg}$ orally 3 times a day for days $1-3$, idarubicin $12 \mathrm{mg} / \mathrm{m}^{2}$ for days $4-6$, and cytarabine $1.5 \mathrm{~g} / \mathrm{m}^{2}$ for days $4-7$. Patients in remission were treated with 5 cycles of consolidation therapy and maintenance therapy with single-agent vorinostat for 12 months. The results showed that $39 \%$ were cytogenetically normal, and 15\% had FLT3 internal tandem duplication (ITD). At the same time, no excess vorinostat-related toxicity was observed. The event-free survival (EFS) was 47 weeks (range 3-134 weeks), and OS was 82 weeks. ORR was $85 \%$, CR rate was $76 \%$, and CR rate with incomplete platelet recovery was 9\%. In diploid patients and patients with FLT3-ITD, ORR was $93 \%$ and $100 \%$, respectively. Thus, the combination of vorinostat with "IA regimen" is safe and active in AML. ${ }^{26}$

Other HDAC inhibitors, including pracinostat (Medchem Express, USA), panobinostat (Medchem Express, USA), and entinostat (Medchem Express, USA), have also shown a great promise in different ways. For example, pracinostat, which is an orally bioavailable HDAC inhibitor, was found to be effective in patients with JAK2 inhibitor (pacritinib [Medchem Express, USA]) in a preclinical study. ${ }^{27}$

\section{Proteasome inhibitor}

In recent years, proteasome inhibitors, especially bortezomib (BSP, Italy), have shown promising activity against leukemias. ${ }^{28-30}$ These inhibitors appear to sensitize leukemia cells to cytarabine and anthracyclines. ${ }^{31}$ In AML patients with FLT3-ITD, proteasome inhibitors induce the degradation of leukemic cells through autophagy. ${ }^{32}$

\section{Other new cytotoxic agents}

Vosaroxin (Medchem Express, USA) is an anticancer quinolone derivative that inserts into DNA, and like anthracyclines, strongly inhibits DNA topoisomerase II and induces double-stranded DNA breaks. Compared with traditional anthracyclines, it has several advantages. First, vosaroxin is associated with significantly reduced cardiotoxicity because it does not form reactive oxygen species, free radicals, or toxic metabolites. Second, vosaroxin can induce p53-independent apoptosis and is not a substrate of P-glycoprotein. ${ }^{33-36}$ Recently, a Phase III, double-blind, placebo-controlled trial was conducted at 101 international sites. One half of the patients were randomly (by a central interactive voice system with a permuted block procedure stratified by geographical location, age, and disease status) assigned to vosaroxin $\left(90 \mathrm{mg} / \mathrm{m}^{2}\right.$ infusion on days 1 and 4 in the first cycle, and $70 \mathrm{mg} / \mathrm{m}^{2}$ in subsequent cycles) plus cytarabine ( $1 \mathrm{~g} / \mathrm{m}^{2}$ infusion for days $\left.1-5\right)$, and other half to placebo plus cytarabine. In the final analysis, median OS was 7.5 months in the vosaroxin plus cytarabine group and 6.1 months in the other group (hazard ratio 0.87 , 95\% CI 0.73-1.02; unstratified log-rank $p=0.061$; stratified $\log$-rank $p=0.024)$. A higher CR rate was indicated in the vosaroxin plus cytarabine group (30\% versus $16 \%, p<0.0001) .{ }^{37}$

In addition, CPX-351 (Jazz, Ireland) is also a nanoscale liposome which has a fixed molar ratio $(5: 1)$ of cytarabine 
and daunorubicin. ${ }^{38}$ This liposome was proved to have an optimal combination, maximizing synergy, and avoiding antagonism. Clinical trials have shown that it has obvious advantage in older patients with secondary AML (sAML, that is, therapy-related AML or AML with a history of antecedent hematologic disorder). A randomized, multicenter, open-label, Phase III study compared first-line CPX-351 $\left(100 \mathrm{U} / \mathrm{m}^{2}\right)$ with daunorubicin $\left(60 \mathrm{mg} / \mathrm{m}^{2}\right)$ plus cytarabine $\left(100 \mathrm{mg} / \mathrm{m}^{2}\right)$ in 309 older patients $(60-75$ years old) with high-risk sAML. The results indicated that CPX-351 group achieved better OS (median 9.56 versus 5.95 months, $p=0.005)$, EFS $(p=0.021)$, and composite CR rates $(47.7 \%$ versus $33.3 \%, p=0.016) .{ }^{39}$

\section{New regimens for the refractory/ relapsed $A M L$ HAA regimen}

Fan et al tested the efficacy and safety of the HAA regimen (homoharringtonine [Harbin Sanjing, China], cytarabine, and aclarubicin [Biovision, USA]) as salvage chemotherapy in the treatment of refractory/relapsed AML (rrAML). They retrospectively analyzed 64 patients with rAML who received the HAA regimen as salvage chemotherapy. The overall CR rate was $70.1 \%$, and $67.1 \%$ of the patients attained $\mathrm{CR}$ after the first induction course. The early death rate was $0 \%$. The median follow-up time was 61 months (range 6-120 months). The estimated 3-year OS rate was $46.8 \%$, and the estimated 3 -year RFS rate was $42.8 \%$. The CR rates of patients with favorable-/intermediate-risk and unfavorable-risk cytogenetics were $76.4 \%$ and $33.3 \%$, respectively. The 3 -year OS rate of favorable-/intermediate-risk and unfavorable-risk group was $53.7 \%$ and $10.0 \%$, respectively. The median survival time of unfavorable-risk group was only 8 months. The side effects associated with the HAA regimen were tolerable, among which the most common toxicities were myelosuppression and infection. ${ }^{40}$

\section{FLAG regimen}

Alwan et al evaluated the "FLAG regimen" (fludarabine, high-dose cytarabine, and G-CSF) in refractory/relapsed AL (rrAL) patients. A prospective study was conducted at the National Center of Hematology and hematology unit of Baghdad Teaching Hospital from July 2008 to July 2010. Twenty rrAL patients ( 5 with refractory acute lymphoblastic leukemia [ALL], 4 with relapsed ALL, 8 with refractory AML, and 3 with relapsed $\mathrm{AML}$ ) received the regimen consisting of fludarabine $30 \mathrm{mg} / \mathrm{m}^{2}$ and cytosine arabinoside $2 \mathrm{~g} / \mathrm{m}^{2}$ for 5 days, and G-CSF $300 \mathrm{mg} / \mathrm{d}$ from day 0 till neutrophil recovery (absolute neutrophil count $>1.0 \times 10^{9} / \mathrm{L}$ ).
A bone marrow examination was conducted on day 30 postchemotherapy to evaluate the response. The results showed that $45 \%$ of patients achieved CR, while $15 \%$ died due to post-chemotherapy complications. Major complications encountered were anemia, fever, bleeding, mucositis, and bacterial infections. ${ }^{41}$

\section{FLANG regimen}

Mehrzad et al evaluated the mortality and response rate of "FLANG regimen". They enrolled 25 rrAL patients aged from 15 to 55 years during 2008-2009. Following 1 cycle, they evaluated the responsiveness to treatment using the bone marrow samples from patients, and followed the patients for a year. The results showed that out of the 25 patients, $40 \%$ responded to treatment. Among the 10 responders, 5 achieved successful bone marrow transplantation (BMT). On the other hand, 13 (52\%) patients who had not achieved CR died during the follow-up. ${ }^{42}$

\section{FCE regimen}

Aldoss et al retrospectively analyzed the outcome of 20 consecutive subjects with rrAML (9 refractory and 11 relapsed) treated with "FCE regimen", consisting of fludarabine, cytarabine, and etoposide (Hengrui, China). Of 20 patients, $75 \%$ achieved CR/CRi. The rate of 4 - and 8-week treatmentrelated mortality (TRM) in all patients during reinduction was $0 \%$ and $5 \%$, respectively. Fifty-three percent of rrAML patients who successfully achieved CR were able to undergo allo-HSCT with a $0 \%$ non-relapse mortality rate. This new regimen also brings a new hope for the salvage treatment of $\operatorname{rrAML} .{ }^{43}$

\section{Decitabine combined with DAG regimen}

Hao et al also compared the clinical safety and efficacy of different chemotherapeutic regimens in the treatment of rrAML. Sixty-seven rrAML patients were enrolled from September 2008, to April 2013, and the regimen consisted of decitabine combined with DAG regimen, CAG regimen, or " $3+7$ " regimen randomly. The results showed that of 19 members in decitabine group, 5 (26.3\%) achieved CR and $4(21.1 \%)$ achieved partial remission (PR) with an ORR of $47.4 \%$. Of 26 patients in CAG regimen group, 8 (30.8\%) achieved CR and 1 (3.8\%) achieved PR, with an ORR of $34.6 \%$. Among 22 patients in " $3+7$ " regimen group, 4 (18.2\%) achieved CR with an ORR of $18.2 \%$. Obviously, the ORR of decitabine group was higher than the other 2 groups $(p<0.05)$. It was noticeable that marrow blast counts were lower in CR patients compared with those in non-CR patients $(p<0.05)$ in the decitabine group, while this difference was 
not observed in " $3+7$ " group and CAG regimen group. Adverse events were similar in the 3 groups, which mainly included myelosuppression, pulmonary infection, nausea, vomiting, and liver dysfunction, and all of them could be well tolerated. The median OS of decitabine group, CAG regimen group, and " $3+7$ ” group after relapse was $7.5,4$, and 3 months, while significant difference was observed between decitabine group and " $3+7$ " regimen group $(p<0.05) .{ }^{44}$

\section{Targeted therapy}

During the last decade, genomic studies of hematologic malignancies have identified a spectrum of recurrent somatic alterations that contribute to AML initiation and maintenance, and which confer sensitivities to molecularly targeted therapies. ${ }^{45,46}$ Some of these new targeted inhibitor drugs have increased the efficacy of the treatment of AML.

\section{FLT3 inhibitors}

FLT3 is the most frequently (approximately 25\%-30\%) mutated gene in AML, and leads to poor prognosis because it can mediate resistance to AML cell death through 3 main signaling pathways, including Ras/MAPK, JAK/p-Stat5, and PI3K-Akt. ${ }^{47,48}$ The most common activating FLT3 mutation is an ITD in the juxtamembrane domain (FLT3-ITD). ${ }^{49,50}$

FLT3 inhibitors can be regarded as the largest family in the targeted agents. These are divided into 2 major categories. First-generation drugs include agents like sorafenib (Bayer, Germany), lestaurtinib (ApexBio, USA), midostaurin (AbMole, USA), sunitinib (ApexBio, USA), and tandutinib (Medchem Express, USA). Sorafenib, which is used widely, has been shown to play an important role in treating rrAML with FLT3 mutations. Giri et al conducted a study on older patients with relapsed FLT3-positive AML using the combination of sorafenib with cytarabine and idarubicin, and found that their disease was controlled for up to 7 months. ${ }^{51}$ $\mathrm{Gu}$ et al also analyzed the efficacy of sorafenib in FLT3mutated AML patients. ${ }^{52}$ Thirty-two cases who were refractory to chemotherapy or relapsed were treated with sorafenib alone or sorafenib in combination with chemotherapy. Ten patients who relapsed after allo-HSCT were retreated with sorafenib alone or sorafenib in combination with donor lymphocyte infusion or chemotherapy. The OS rate was $73.8 \% ; 4(9.5 \%)$ achieved complete molecular remission (CMR), 9 (21.4\%) achieved CR, 8 (19\%) achieved CR with CRi, 10 (23.8\%) achieved PR, and 11 (26.2\%) achieved no remission (NR). The response rate of sorafenib alone for 17 patients was $70.6 \%$, and that of sorafenib plus chemotherapy was $66.7 \%(p=0.555)$. Among the 13 patients who received allo-HSCT, 6 achieved CMR/CR/CRi, 4 achieved
PR, and 3 achieved NR before transplant. Walker et al conducted a Phase I study of the midostaurin with bortezomib alone and in combination with chemotherapy in patients with AML. ${ }^{53}$ Patients on dose level (DL) 1 and DL2 received midostaurin $50 \mathrm{mg}$ twice a day orally and escalating doses of bortezomib (from 1 to $1.3 \mathrm{mg} / \mathrm{m}^{2}$ ), while patients on DL3 or higher received midostaurin combination with bortezomib following chemotherapy with mitoxantrone, etoposide, and cytarabine. The results indicated that none of patients on DL1 and DL2 achieved dose-limiting toxicities (DLTs) or clinical responses. However, among patients enrolled in the DL3 or higher group, DLTs were reflected in peripheral neuropathy, and cardiovascular and digestive system disorders. Overall, a CR rate of $56.5 \%$ and an OS rate of $82.5 \%$ were observed in the clinical trial. Sunitinib was also added to chemotherapy in older patients with FLT3-mutated AML, and data showed the CR rate was $53 \%$ and $71 \%$ in patients with FLT3-ITD and FLT3-TKD mutations, respectively. During the follow-up period, 13 patients who achieved CR underwent a consolidated therapy with high-dose cytarabine, and 7 of them received sunitinib maintenance therapy. The median OS in this study was 18.8 months. ${ }^{54}$

The second-generation agents, promising to have better potency and cause less side effects, include crenolanib, quizartinib (Selleck, USA), and cabozantinib (Medchem Express, USA). Cooper et al concluded quizartinib (AbMole, USA) (60 mg/m² daily) plus intensive chemotherapy achieved a near-complete inhibition of FLT3 phosphorylation in all patients. ${ }^{55}$ However, the major challenge in the use of FLT3 inhibitors is the drug resistance. The point mutations that lead to resistance include N676, F691, and D835, together with FLT3-ITD. ${ }^{56}$ The new FLT3 inhibitors, G-749 and ASP2215, have been proved to cause strong inhibition of FLT3 phosphorylation and increase the ability to overcome drug resistance in preclinical trials, but further studies are needed to evaluate their clinical efficacy. ${ }^{57,58}$

\section{Isocitrate dehydrogenase (IDH) inhibitors}

IDH1 and IDH2 mutations occur in up to $20 \%$ of AML patients with normal karyotype. The key pharmacological activity of IDH1 and IDH2 is that they can convert isocitrate to $\alpha$-ketoglutarate $(\alpha-K G)$ as metabolic enzymes. Thus, IDH1 and IDH2 mutations are novel gain-of-function mutations, which carry a neomorphic enzyme to catalyze the conversion of $\alpha-K G$ to the oncometabolite 2-hydroxyglutarate. This process results in the competitive inhibition of $\alpha-\mathrm{KG}$ dependent enzymes, including TET2, impaired hematopoietic differentiation, and hypermethylation of target genes. ${ }^{59}$ The IDH1 inhibitor, AG-120 (Selleck, USA), and the IDH2 
inhibitor, AG-221 (Selleck, USA), have been demonstrated to be significantly effective in the treatment of AML in 2 separate clinical trials. In a study of AG-120, it was reported that effective response was found in 16 of 52 (31\%) patients, and the CR rate was $16 \% .{ }^{60}$ Similarly, in a Phase I study, 158 patients with IDH2-mutated AML were treated once or twice a day with AG-221 at a dose ranging from 30 to $200 \mathrm{mg}$. The ORR was $40 \%$, including a CR rate of $16.5 \% .{ }^{61}$ Based on these data and because the drugs are well tolerated, the FDA has approved these medications with an orphan drug designation for treatment of the patients with AML.

\section{Nuclear exporter inhibitors}

CRM1/XPO1 (Imgenex, USA) is a key nuclear export protein which shows inhibitory effects on the nuclear accumulation of tumor suppressor proteins and renders cancer cells susceptible to apoptosis. Selinexor is orally bioavailable and belongs to a novel class of small-molecule compounds with activity against a wide variety of cancers. ${ }^{62}$ Selinexor (Amquara, USA) is currently studied in a Phase I clinical study on hematologic malignancies (ClinicalTrials.gov NCT0167892). In addition, many Phase I/II clinical trials are currently ongoing to assess the safety, tolerability, and activity of selinexor in AML patients. ${ }^{63}$

\section{Immunotherapy}

Novel antibody therapies are new revolutionaries in AML treatment. Some advances in these therapies in the past 5 years are described below.

\section{Gemtuzumab ozogamicin (GO)}

GO is a monoclonal antibody (Pfizer, USA) against CD33 cell adhesion molecule, which is differentially expressed during myeloid differentiation. Borthakur et al conducted a study on a new regimen (FLAG-GO) including fludarabine $\left(30 \mathrm{mg} / \mathrm{m}^{2}\right)$, cytarabine $\left(2 \mathrm{~g} / \mathrm{m}^{2}\right)$, G-CSF, and low-dose GO $\left(3 \mathrm{mg} / \mathrm{m}^{2}\right.$ infusion over 2 hours on day 1 ) as a preference therapy core binding factor (CBF) AML, in which 45 patients (median age 48 years) were enrolled. The remission rate was $95 \%$, with $5 \%$ of induction deaths. The OS and RFS probabilities at 3 years were $78 \%$ and $85 \%$, respectively. "FLAG-GO regimen" worked well in CBF-AML patients. ${ }^{64}$ However, the agent was approved by the FDA in 2000 for the treatment of elderly patients with relapsed CD33-positive AML at a dose of $9 \mathrm{mg} / \mathrm{m}^{2}$ for 2 days 2 weeks apart. Almost at once, its safety became a focus, and it was found that the agent triggers a particular liver signal and it was finally withdrawn from practice in 2010. The significant outcome of this agent in the treatment of AML has been recognized by most of the hematologists. In this review, we focus on its safe dose and the balance between its efficacy and side effects. Parigger et al reviewed 14 studies of GO used in AML treatment, and then drew scatter plots and linear regressions to evaluate the relationship between the dose of GO and its toxicity and efficacy. Results showed that a nonsignificant increase in bilirubin level and the incidence of veno-occlusive disease was seen with higher doses of single-agent GO. On the other hand, even a low dose of $3 \mathrm{mg} / \mathrm{m}^{2}$ can have an antileukemic effect, but available data do not allow conclusions on its dose dependency. Unfortunately, results of an analysis indicated that higher doses of GO account for more adverse events. ${ }^{65}$

\section{Other monoclonal antibodies}

SGN-33A is a humanized anti-CD33 monoclonal antibody conjugated to a potent DNA-cross-linking toxin, which is a substrate of drug-efflux enzymes. This novel agent carries a pyrrolobenzodiazepine (PBD) dimer. Molecules in the PBD family cause cell death by cross-linking with DNA and interrupting cell division and are being explored clinically for the treatment of human cancers. ${ }^{66-68}$ Studies $^{69}$ have showed that the cytotoxic effects of SGN-33A involve DNA damage with ensuing cell cycle arrest and apoptotic cell death. A previous study demonstrated that SGN-33A had more potent antileukemic activity than $\mathrm{GO}$ in primary diagnosed patient samples, cell lines, and xenograft model. ${ }^{69}$ Besides, SGN-33A is currently under investigation as a single agent and in combination with other drugs in several clinical settings (NCT02326584). In a Phase I dose escalation trial, 38 patients with relapsed AML were treated with SGN-33A (range 5-60 $\mu \mathrm{m} / \mathrm{kg}$ infusion every 3 weeks), of which 16 (42\%) had clearance of marrow blasts and showed good tolerance. ${ }^{70}$ Clinical trials on other monoclonal antibodies such as AMG 330 (antiCD33 and CD3), CD45, CD66, and CD123 are underway. ${ }^{71}$

\section{Chimeric antigen receptor (CAR) T-cell therapy}

Among the immunotherapies, CAR T-cell therapy has shown a great promise for the treatment of hematologic malignancies, especially B-cell malignancies, in recent years. CAR T-cells are redirected $\mathrm{T}$-cells that can recognize cancer antigens in a major histocompatibility complex-independent manner. It comprises 2 main functional domains: an extracellular antigen recognition domain, called a single-chain variable fragment, and an intracellular signaling domain. ${ }^{72}$ However, its efficacy in AML is still not confirmed. Jetani et al proved the efficacy of CAR T-cell therapy in FLT3-mutated AML 
patients this year. They engineered $\mathrm{CD}^{+}$and $\mathrm{CD}^{+}{ }^{+} \mathrm{T}$-cells expressing an FLT3-specific CAR and demonstrated that the T-cells conferred potent reactivity against AML cell lines and primary AML blasts that expressed either wild-type FLT3 or FLT3-ITD. Also, their data provided the first proof of concept that CAR T-cell immunotherapy and small-molecule inhibition can be used synergistically, as exemplified by superior antileukemic efficacy of FLT3-CAR T-cells in combination with crenolanib. ${ }^{73}$

\section{Conclusion}

AML is a complex disease with high mortality. The drug treatment methods for AML include chemotherapy, targeted therapy, and immunotherapy. Among the different chemotherapies, the standard " $7+3$ " regimen is the primary choice, but researchers have made significant improvements in this regimen based on patients' conditions. Meanwhile, new drugs are rapidly expanding with increased understanding of the biology and molecular landscape, such as emerging cytotoxic chemotherapies, targeted small-molecule inhibitors, and monoclonal antibodies. HMAs can be used in older patients due to their low toxicity. New nucleoside analogs, such as clofarabine and sapacitabine, have also been studied. Clofarabine, as monotherapy or in combination with cytarabine, has achieved satisfying outcome in clinical trials, while sapacitabine needs further research for use in clinical treatment. Vorinostat, a new HDAC agent, when combined with "IA regimen," is safe and active in AML treatment. Vosaroxin, a kind of anticancer quinolone derivative, has showed its superiority to traditional anthracyclines, with respect to cardiotoxicity and induction of p53-independent apoptosis. Other new cytotoxic agents, such as bortezomib and CPX-351, also play a role in AML therapy. With the emergence of resistance to different drugs, researchers have started to search for new regimens for rrAML, including HAA, FLAG, FLANG, FEC, and decitabine and DAG, all of which are effective to some extent. There are also improvements made in therapies for patients with different mutations, such as FLT3 and IDH. In FLT3-mutated group, first-generation agents such as sorafenib, lestaurtinib, midostaurin, sunitinib, and tandutinib are used, while in the IDH-mutated group, AG-120 and AG-211 are used. Among immunotherapies, GO, especially the FLAG-GO regimen, plays an important role. Although CAR T-cell therapy is mainly used in B-cell tumor, it also holds promise for FLT3mutated AML, and FLT3-CAR T-cells in combination with crenolanib have shown superior antileukemic efficacy.

However, a large number of clinical trials are needed to improve the use of these drugs in AML patients with less toxicity and improved efficacy, and more studies should be devoted to better understand the action of these new drugs which will lead to their safe and regular use.

\section{Acknowledgments}

This review was supported by the Key Medical Subjects of Jiangsu Province (2012-12), Natural Science Foundation of Jiangsu Province, and Technology Support Program of Jiangsu Province, and the key projects in Jiangsu Province Department of Medicine (BL2014078), which are hosted by Professor Baoan Chen.

\section{Disclosure}

The authors report no conflicts of interest in this work.

\section{References}

1. Siegel RL, Miller KD, Jemal A. Cancer statistics, 2017. CA Cancer J Clin. 2017;66(1):7-30.

2. National Comprehensive Cancer Network. NCCN clinical practice guidelines in oncology: antiemesis, V3.2017. Available from: http:// www.nccn.org/professionals/physician_gls/PDF/antiemesis.pdf. Accessed February 22, 2017.

3. Padron E, Fernandez H. Anthracycline dose intensification in young adults with acute myeloid leukemia. Ther Adv Hematol. 2012;3(1): $17-27$.

4. Holowiecki J, Grosicki S, Giebel S, et al. Cladribine, but not fludarabine, added to daunorubicin and cytarabine during induction prolongs survival of patients with acute myeloid leukemia: a multicenter, randomized phase III study. J Clin Oncol. 2012;30(20):2441-2448.

5. Willemze R, Suciu S, Meloni G, et al. High-dose cytarabine in induction treatment improves the outcome of adult patients younger than age 46 years with acute myeloid leukemia: results of the EORTC-GIMEMA AML-12 trial. J Clin Oncol. 2014;32(3):219-228.

6. Arellano M, Winton E, Pan L, et al. High-dose cytarabine induction is well tolerated and active in patients with de novo acute myeloid leukemia older than 60 years. Cancer. 2012;118(2):428-433.

7. Burnett AK, Russell NH, Hills RK, et al. Optimization of chemotherapy for younger patients with acute myeloid leukemia: results of the medical research council AML15 trial. J Clin Oncol. 2013;31(27): 3360-3368.

8. Herman JG, Baylin SB. Gene silencing in cancer in association with promoter hypermethylation. N Engl J Med. 2003;349(21):2042-2054.

9. Gore SD, Baylin S, Sugar E, et al. Combined DNA methyltransferase and histone deacetylase inhibition in the treatment of myeloid neoplasms. Cancer Res. 2006;66(12):6361-6369.

10. Qiu X, Hother C, Ralfkiær UM, et al. Equitoxic doses of 5-azacytidine and 5-aza-2'deoxycytidine induce diverse immediate and overlapping heritable changes in the transcriptome. PLoS One. 2010;5(9):e12994.

11. Almstedt M, Blagitko-Dorfs N, Duque-Afonso J, et al. The DNA demethylating agent 5-aza-2'-deoxycytidine induces expression of NY-ESO-1 and other cancer/testis antigens in myeloid leukemia cells. Leuk Res. 2010;34(7):899-905.

12. Quintás-Cardama A, Ravandi F, Liu-Dumlao T, et al. Epigenetic therapy is associated with similar survival compared with intensive chemotherapy in older patients with newly diagnosed acute myeloid leukemia. Blood. 2012;120(24):4840-4845.

13. Fenaux P, Mufti GJ, Hellstrom-Lindberg E, et al. Efficacy of azacitidine compared with that of conventional care regimens in the treatment of higher-risk myelodysplastic syndromes: a randomised, open-label, Phase III study. Lancet Oncol. 2009;10(3):223-232. 
14. Fenux P, Mufti GJ, Hellstrom-Lindberg E, et al. Azacitidine prolongs overall survival compared with conventional care regimens in elderly patients with low bone marrow blast count acute myeloid leukemia. J Clin Oncol. 2010;28(4):562-569.

15. Cashen AF, Schiller GJ, O’Donnell MR, DiPersio JF. Multicenter, Phase II study of decitabine for the first-line treatment of older patients with acute myeloid leukemia. J Clin Oncol. 2010;28(4):556-561.

16. Issa JP, Garcia-Manero G, Giles FJ, et al. Phase 1 study of low-dose prolonged exposure schedules of the hypomethylating agent 5-aza-2'deoxycytidine (decitabine) in hematopoietic malignancies. Blood. 2004; 103(5):1635-1640.

17. Kantarjian HM, Thomas XG, Dmoszynska A, et al. Multicenter, randomized, open-label, Phase III trial of decitabine versus patient choice, with physician advice, of either supportive care or low-dose cytarabine for the treatment of older patients with newly diagnosed acute myeloid leukemia. J Clin Oncol. 2012;30(21):2670-2677.

18. Issa PJ, Roboz G, Rizzieri D, et al. Safety and tolerability of guadecitabine (SGI-110) in patients with myelodysplastic syndrome and acute myeloid leukaemia: a multicentre, randomised, dose-escalation Phase 1 study. Lancet Oncol. 2015;16(9):1099-1110.

19. Kantarjian HM, Erba HP, Claxton D, et al. Phase II study of clofarabine monotherapy in previously untreated older adults with acute myeloid leukemia and unfavorable prognostic factors. J Clin Oncol. 2010;28(4): 549-555.

20. Takahashi K, Kantarjian H, Garcia-Manero G, et al. Clofarabine plus low-dose cytarabine is as effective as and less toxic than intensive chemotherapy in elderly AML patients. Clin Lymphoma Myeloma Leuk. 2016;16(3):163-168.

21. Azuma A, Huang P, Matsuda A, Plunkett W. 2'-C-cyano-2'-deoxy1-beta-D-arabinopentofuranosylcytosine: a novel anticancer nucleoside analog that causes both DNA strand breaks and $\mathrm{G}(2)$ arrest. Mol Pharmacol. 2001;59(4):725-731.

22. Norkin M, Richards AI. Sapacitabine in the treatment of acute myeloid leukemia. Expert Rev Anticancer Ther. 2015;15(11):1-6.

23. Kantarjian H, Faderl S, Garcia-Manero G, et al. Oral sapacitabine for the treatment of acute myeloid leukaemia in elderly patients: a randomised phase 2 study. Lancet Oncol. 2012;13(11):1097-1104.

24. Green SR, Choudhary AK, Fleming IN. Combination of sapacitabine and HDAC inhibitors stimulates cell death in AML and other tumour types. Br J Cancer. 2010;103(9):1391-1399.

25. Pastore F, Levine RL. Epigenetic regulators and their impact on therapy in acute myeloid leukemia. Haematologica. 2016;101(3):269-278.

26. Garcia-Manero G, Tambaro FP, Bekele NB, et al. Phase II trial of vorinostat with idarubicin and cytarabine for patients with newly diagnosed acute myelogenous leukemia or myelodysplastic syndrome. J Clin Oncol. 2012;30(18):2204-2210.

27. Novotnydiermayr V, Hart S, Goh KC, et al. The oral HDAC inhibitor pracinostat (SB939) is efficacious and synergistic with the JAK2 inhibitor pacritinib (SB1518) in preclinical models of AML[J]. Blood Cancer Journal. 2012;2(5):e69.

28. Houghton PJ, Morton CL, Kolb EA, et al. Initial testing (stage 1) of the proteasome inhibitor bortezomib by the pediatric preclinical testing program. Pediatr Blood Cancer. 2008;50(1):37-45.

29. Attar EC, De Angelo DJ, Supko JG, et al. Phase I and pharmacokinetic study of bortezomib in combination with idarubicin and cytarabine in patients with acute myelogenous leukemia. Clin Cancer Res. 2008;14(5):1446-1454.

30. Attar EC, Johnson JL, Amrein PC, et al. Bortezomib added to daunorubicin and cytarabine during induction therapy and to intermediate-dose cytarabine for consolidation in patients with previously untreated acute myeloid leukemia age 60 to 75 years: CALGB (Alliance) study 10502. J Clin Oncol. 2013;31(7):923-929.

31. Horton TM, Perentesis JP, Gamis AS, et al. A Phase 2 study of bortezomib combined with either idarubicin/cytarabine or cytarabine/etoposide in children with relapsed, refractory or secondary acute myeloid leukemia: a report from the children's oncology group. Pediatr Blood Cancer. 2014;61(10):1754-1760.
32. Larrue C, Saland E, Boutzen $\mathrm{H}$, et al. Proteasome inhibitors induce FLT3-ITD degradation through autophagy in AML cells. Blood. 2016; 127(7):882-892.

33. Evanchik MJ, Allen D, Yoburn JC, Silverman JA, Hoch U. Metabolism of (+)-1,4-dihydro-7-(trans-3-methoxy-4-methylamino-1-pyrrolidinyl)4-oxo-1-(2-thiaz olyl)-1,8-naphthyridine-3-carboxylic acid (voreloxin; formerly SNS-595), a novel replication dependent DNA-damaging agent. Drug Metab Dispos. 2009;37(3):594-601.

34. Hawtin RE, Stockett DE, Byl JA, et al. Voreloxin is an anticancer quinolone derivative that intercalates DNA and poisons topoisomerase II. PLoS One. 2010;5(4):e10186.

35. Mjos KD, Cawthray JF, Jamieson G, Fox JA, Orvig C. Iron(III)-binding of the anticancer agents doxorubicin and vosaroxin. Dalton Trans. 2015; 44(5):2348-2358.

36. Walsby EJ, Coles SJ, Knapper S, Burnett AK. The topoisomerase II inhibitor voreloxin causes cell cycle arrest and apoptosis in myeloid leukemia cells and acts in synergy with cytarabine. Haematologica. 2011; 96(3):393-399.

37. Ravandi F, Ritchie EK, Sayar H, et al. Vosaroxin plus cytarabine versus placebo plus cytarabine in patients with first relapsed or refractory acute myeloid leukaemia (VALOR): a randomised, controlled, double-blind, multinational, phase 3 study. Lancet Oncol. 2015;16(9): 1025-1036.

38. Feldman EJ, Lancet JE, Kolitz JE, et al. First-in-man study of CPX351: a liposomal carrier containing cytarabine and daunorubicin in a fixed 5:1 molar ratio for the treatment of relapsed and refractory acute myeloid leukemia. J Clin Oncol. 2011;29(8):979-985.

39. Lancet JE, Cortes JE, Hogge DE, et al. Phase 2 trial of CPX-351, a fixed 5:1 molar ratio of cytarabine/daunorubicin, vs cytarabine/daunorubicin in older adults with untreated AML. Blood. 2014;123(21):3239-3246.

40. Fan $\mathrm{CH}, \mathrm{Yu}$ WJ, Mai WY, et al. [Efficacy of HAA regimen in the treatment of 64 patients with refractory/relapsed acute myeloid leukemia]. Zhonghua Xue Ye Xue Za Zhi. 2016;37(2):100-104. Chinese [with English abstract].

41. Alwan AF, Matti BF, Naji AS, Jawad AM. The efficacy of fludarabine, high dose cytosine arabinoside with granulocyte colony stimulating factor (FLAG) protocol as salvage therapy for refractory/relapsed acute leukemias in adult Iraqi patients. Indian J Hematol Blood Transfus. 2014;30(4):231-235.

42. Mehrzad VA, Liaghat L, Ashrafi F, Tazhibi M, Hajalikhani M, Alijanian N. The mortality and response rate after FLANG regimen in patients with refractory/relapsed acute leukemia. Adv Biomed Res. 2012;1:54.

43. Aldoss I, Ji L, Haider M, Pullarkat V. The combination of fludarabine, cytarabine and etoposide is an active and well-tolerated regimen in relapsed/refractory acute myeloid leukemia. Acta Haematol. 2014; 131(4):201-207.

44. Hao J, Wang L, Wang Y, et al. [Comparative analysis of decitabine combined with DAG regimen and other regimens in treatment of refractory/relapsed acute myeloid leukemia]. Zhonghua Xие Ye Xиe Za Zhi. 2014;35(6):481-485. Chinese [with English abstract].

45. Lindsley RC, Mar BG, Mazzola E, et al. Acute myeloid leukemia ontogeny is defined by distinct somatic mutations. Blood. 2015;125(9): $1367-1376$.

46. Gaidzik V, Döhner K. Prognostic implications of gene mutations in acute myeloid leukemia with normal cytogenetics. Semin Oncol. 2008; 35(4):346-355.

47. Mizuki M, Fenski R, Halfter H, et al. Flt3 mutations from patients with acute myeloid leukemia induce transformation of 32D cells mediated by the Ras and STAT5 pathways. Blood. 2000;96(12):3907-3914.

48. Choudhary C, Schwäble J, Brandts C, et al. AML-associated Flt3 kinase domain mutations show signal transduction differences compared with Flt3 ITD mutations. Blood. 2005;106(1):265-273.

49. Moreno I, Martín G, Bolufer P, et al. Incidence and prognostic value of FLT3 internal tandem duplication and D835 mutations in acute myeloid leukemia. Haematologica. 2003;88(1):19-24. 
50. Yamamoto Y, Kiyoi H, Nakano Y, et al. Activating mutation of D835 within the activation loop of FLT3 in human hematologic malignancies. Blood. 2001;97(8):2434-2439.

51. Giri S, Hamdeh S, Bhatt VR, Schwarz JK. Sorafenib in relapsed AML with FMS-like receptor tyrosine kinase-3 internal tandem duplication mutation. J Natl Compr Canc Netw. 2015;13(5):508-514.

52. Gu B, Chen GH, Shen HJ, et al. [Improved clinical outcome of acute myeloid leukemia with FLT3-ITD mutation treated with sorafenib]. Zhonghua Nei Ke Za Zhi. 2016;55(4):293-297. Chinese [with English abstract].

53. Walker AR, Wang H, Walsh K, et al. Midostaurin, bortezomib and MEC in relapsed/refractory acute myeloid leukemia. Leuk Lymphoma. 2016;57(9):2100-2108.

54. Fiedler W, Kayser S, Kebenko M, et al. Sunitinib and intensive chemotherapy in patients with acute myeloid leukemia and activating FLT3 mutations: results of the AMLSG 10-07 study (ClinicalTrials.gov No. NCT00783653). In: Proceedings of 56th ASH Annual Meeting and Exposition; December 8-11, 2012; Atlanta, GA.

55. Cooper TM, Cassar J, Eckroth E, et al. A phase I study of quizartinib combined with chemotherapy in relapsed childhood leukemia: a therapeutic advances in childhood leukemia and lymphoma (TACL) study. Clin Cancer Res. 2016;22(16):4014-4022.

56. Smith CC, Wang Q, Chin CS, et al. Validation of ITD mutations in FLT3 as a therapeutic target in human acute myeloid leukemia. Nature. 2012;485(7397):260-263.

57. Lee HK, Kim HW, Lee IY, et al. G-749, a novel FLT3 kinase inhibitor, can overcome drug resistance for the treatment of acute myeloid leukemia. Blood. 2014;123(14):2209-2219.

58. Altman JK, Perl AE, Cortes JE, et al. Antileukemic activity and tolerability of ASP2215 $80 \mathrm{mg}$ and greater in FLT3 mutation-positive subjects with relapsed or refractory acute myeloid leukemia: results from a phase 1/2, open-label, dose-escalation/dose-response study. In: Proceedings of the American Society of Hematology Annual Meeting; December 5-8, 2015; Orlando, FL.

59. Medeiros BC, Fathi AT, DiNardo CD, Pollyea DA, Chan SM, Swords R Isocitrate dehydrogenase mutations in myeloid malignancies. Leukemia. 2017;31(2):272-281.

60. De Botton S, Pollyea DA, Stein EM, et al. Clinical safety and activity of AG-120, a first-in-class, potent inhibitor of the IDH1 mutant protein, in a Phase 1 study of patients with advanced IDH1-mutant hematologic malignancies. Blood. 2015:100704.
61. DiNardo CD, Stein EM, Altman J, et al. AG-221, an oral, selective, firstin-class, potent inhibitor of the IDH2 mutant enzyme, induced durable responses in a Phase I study of IDH2 mutation-positive advanced hematologic malignancies. Blood. 2015:100170.

62. Kojima K, Kornblau SM, Ruvolo V, et al. Prognostic impact and targeting of CRM1 in acute myeloid leukemia. Blood. 2013;121(20): 4166-4174.

63. Ranganathan $\mathrm{P}, \mathrm{Yu} X, \mathrm{Na} \mathrm{C}$, et al. Preclinical activity of a novel CRM1 inhibitor in acute myeloid leukemia. Blood. 2012;120(9):1765-1773.

64. Borthakur G, Cortes JE, Estey EE, et al. Gemtuzumab ozogamicin with fludarabine, cytarabine, and granulocyte colony stimulating factor (FLAG-GO) as front-line regimen in patients with core binding factor acute myelogenous leukemia. Am J Hematol. 2014;89(10):964-968.

65. Parigger J, Zwaan CM, Reinhardt D, Kaspers GJ. Dose-related efficacy and toxicity of gemtuzumab ozogamicin in pediatric acute myeloid leukemia. Expert Rev Anticancer Ther. 2015;16(2):137-146.

66. Hartley JA, Hochhauser D. Small molecule drugs - optimizing DNA damaging agent-based therapeutics. Curr Opin Pharmacol. 2012;12(4): 398-402.

67. Smellie M, Bose DS, Thompson AS, Jenkins TC, Hartley JA, Thurston DE. Sequence-selective recognition of duplex DNA through covalent interstrand cross-linking: kinetic and molecular modeling studies with pyrrolobenzodiazepine dimers. Biochemistry. 2003;42(27):8232-8239.

68. Wu J, Clingen PH, Spanswick VJ, et al. $\gamma$-H2AX foci formation as a pharmacodynamic marker of DNA damage produced by DNA cross-linking agents: results from 2 phase I clinical trials of SJG-136 (SG2000). Clin Cancer Res. 2013;19(3):721-730.

69. Kung Sutherland MS, Walter RB, Jeffrey SC, et al. SGN-CD33A: a novel CD33-targeting antibody-drug conjugate using a pyrrolobenzodiazepine dimer is active in models of drug-resistant AML. Blood. 2013;122(8):1455-1463.

70. Stein EM, Stein A, Walter RB, et al. Interim analysis of a phase 1 trial of SGNCD33A in patients with CD33-positive acute myeloid leukemia (AML). Blood. 2014;124(21):623.

71. Gasiorowski RE, Clark GJ, Bradstock K, Hart DN. Antibody therapy for acute myeloid leukaemia. Br J Haematol. 2014;164(4):481-495.

72. Muhammand N, Mao Q, Xia H. CAR T-cells for cancer therapy. Biotechnol Genet Eng Rev. 2017;33(2):190-226.

73. Jetani H, Garcia-Cadenas I, Nerreter T, et al. CAR T-cells targeting FLT3 have potent activity against FLT3-ITD+ AML and act synergistically with the FLT3-inhibitor crenolanib. Leukemia. Epub 2018 Feb 5.

\section{Publish your work in this journal}

Drug Design, Development and Therapy is an international, peerreviewed open-access journal that spans the spectrum of drug design and development through to clinical applications. Clinical outcomes, patient safety, and programs for the development and effective, safe, and sustained use of medicines are the features of the journal, which

\section{Dovepress}

has also been accepted for indexing on PubMed Central. The manuscript management system is completely online and includes a very quick and fair peer-review system, which is all easy to use. Visit http://www.dovepress.com/testimonials.php to read real quotes from published authors. 